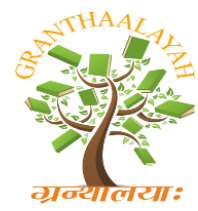

INTERNATIONAL JOURNAL OF RESEARCH GRANTHAALAYAH

A knowledge Repository

Science

\title{
NUMERICAL SOLUTION TO LINEAR SINGULARLY PERTURBED TWO POINT BOUNDARY VALUE PROBLEMS USING B-SPLINE COLLOCATION METHOD
}

\author{
Rajashekhar Reddy .Y *1 \\ ${ }^{* 1}$ Assistant Professor, Department of Mathematics, JNT University College of Engineering \\ Jagitial, Nachupally (kondagattu), Karimnagar-505501,Telangana State, INDIA
}

\begin{abstract}
A Recursive form cubic B-spline basis function is used as basis in B-spline collocation method to solve second linear singularly perturbed two point boundary value problem. The performance of the method is tested by considering the numerical examples with different boundary conditions. Results of numerical examples show the robustness of the method when compared with the analytical solution.
\end{abstract}

Keywords:

Collocation method, B-splines, linear singularly perturbed problem.

Cite This Article: Rajashekhar Reddy .Y, "NUMERICAL SOLUTION TO LINEAR SINGULARLY PERTURBED TWO POINT BOUNDARY VALUE PROBLEMS USING BSPLINE COLLOCATION METHOD” International Journal of Research - Granthaalayah, Vol. 4, No. 1 (2016): 158-164.

\section{INTRODUCTION}

The singularly perturbed two point boundary value problems are governing differential equations to represents the process in the fields such as combustion, chemical reactor theory, nuclear engineering, elasticity, fluid mechanics etc.

In recent years, many numerical methods are developed to solve linear singularly perturbed two point boundary value problem. Researchers presented many numerical methods [13-15] from 2006 to 2013 to deal with one-dimensional singular perturbation problems. These problems considered include linear, non-linear reaction-diffusion, delay differential equations. The numerical techniques reviewed in this survey include finite-difference methods, spline approximation methods and computational methods for boundary value techniques.

However, it is observed from the recent literature that B-spline basis functions are derived using fixed equidistant space for a particular degree only. If the recursive formulation given by Carl. De boor [12] is applied, the basis function evaluation can be generalized and without fixing of 
degree of the basis function can be used in collocation method for uniform or non uniform mesh sizes.

In this paper, after defining the B-spline basis function recursively, the B-spline collocation method is described and formulated. The efficiency of the method is demonstrated using the second order singular differential equations with Neumann's boundary conditions.

Considering second order linear singularly perturbed two point boundary value problem of the form

$$
\varepsilon \frac{d^{2} U}{d x^{2}}+k_{1} P(x) \frac{d U}{d x}+k_{2} Q(x) U=R(x) \quad, \quad a \leq x \leq b \quad \ldots \ldots
$$

With boundary conditions $\quad U(a)+\varepsilon U^{\prime}(a)=d 1, U(b)+\varepsilon U^{\prime}(b)=d 2$ where $a, b, d_{1}, d_{2}, k_{1}$ and $k_{2}$ are constant $P(x), Q(x)$ and $R(x)$ are functions of $x$

Let $\quad U^{h}(x)=\sum_{i=-2}^{n-1} C_{i} N_{i, p}(x) \quad \ldots \ldots$ (2) , where $C_{i}$ 's are constants to be determined and $N_{i, p}(x)$ are B-spline basis functions, be the approximate global solution to the exact solution $U(x)$ of the considered second order singular differential equation (1).

\subsection{B-splines}

In this section, definition and properties of B-spline basis functions [1,2] are given in detail. A zero degree and other than zero degree B-spline basis functions are defined at $x_{i}$ recursively over the knot vector space $X=\left\{x_{1}, x_{2}, x_{3} \ldots \ldots . . x_{n-1}, x_{n}\right\}$ as

i) if $p=0$

$$
\begin{array}{ll}
N_{i, p}(x)=1 & \text { if } \quad x \in\left(x_{i}, x_{i+i}\right) \\
N_{i, p}(x)=0 & \text { if } x \notin\left(x_{i}, x_{i+i}\right)
\end{array}
$$

ii) if $p \geq 1$

$$
N_{i, p}(x)=\frac{x-x_{i}}{x_{i+p}-x_{i}} N_{i, p-1}(x)+\frac{x_{i+p+1}-x}{x_{i+p+1}-x_{i+1}} N_{i+1, p-1}(x)
$$

where $\mathrm{p}$ is the degree of the $\mathrm{B}$-spline basis function and $x$ is the parameter belongs to $X$.When evaluating these functions, ratios of the form $0 / 0$ are defined as zero.

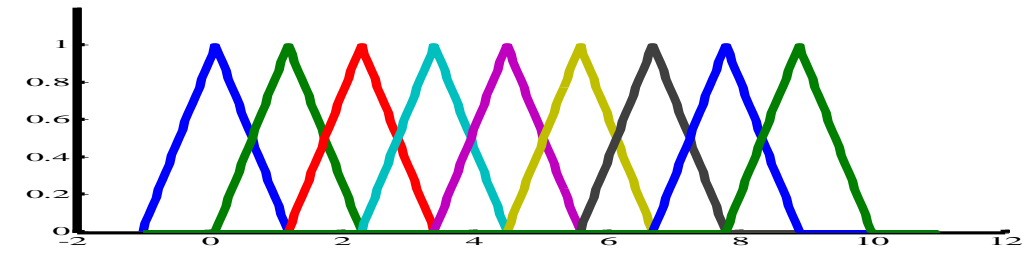

Figure 1: First degree B-spline basis function with uniform Knot vector $\mathrm{X}=\{-1,0,1,2,3,4,5,6,7,8,9,10,11,12\}$ 


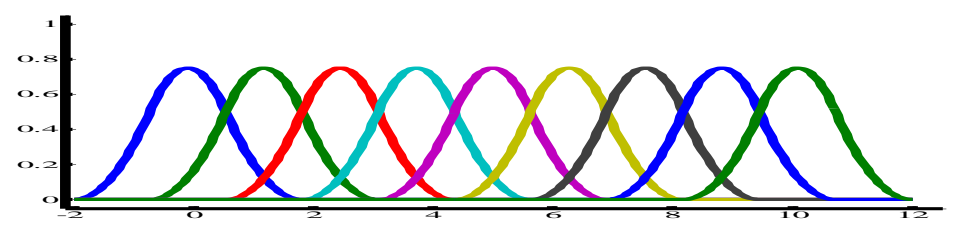

Figure 2: Second degree B-spline basis function with uniform Knot vector $\mathrm{X}=\{-2,-1,0,1,2,3,4,5,6,7,8,9,10,11,12\}$

\subsection{Derivatives of B-splines}

$$
\begin{aligned}
& \text { If } \quad \mathrm{p}=2, \\
& N_{i, p}^{\prime}(x)=\frac{x-x_{i}}{x_{i+p}-x_{i}} N_{i, p-1}^{\prime}(x)+\frac{N_{i, p-1}(x)}{x_{i+p}-x_{i}}+\frac{x_{i+p+1}-x}{x_{i+p+1}-x_{i+1}} N_{i+1, p-1}^{\prime}(x)-\frac{N_{i+1, p-1}(x)}{x_{i+p+1}-x_{i+1}} \\
& N^{\prime \prime} i, p(x)=2 \frac{N_{i, p-1}^{\prime}(x)}{x_{i+p}-x_{i}}-2 \frac{N^{\prime} i+1, p-1(x)}{x_{i+p+1}-x_{i+1}}
\end{aligned}
$$

In the above equations, the basis functions are defined as recursively in terms of previous degree basis function i.e. the $\mathrm{p}^{\text {th }}$ degree basis function is the combination of ratios of knots and (p-1) degree basis function. Again (p-1) ${ }^{\text {th }}$ degree basis function is defined as the combination ratios of knots and ( $\mathrm{p}-2)$ degree basis function. In a similar way every B-spline basis function of degree up to (p-(p-2)) is expressed as the combination of the ratios of knots and its previous B-spline basis functions.

The B-spline basis functions are defined on knot vectors. Knots are real quantities. Knot vector is a non decreasing set of Real numbers. Knot vectors are classified as non-uniform knot vectors, uniform knot vector and open uniform knot vectors. Uniform knot vector in which difference of any two consecutive knots is constant is used for test problems in this paper. Two knots are required to define the zero degree basis function. In a similar way, a $\mathrm{p}^{\text {th }}$ degree B-spline basis function at a knot have a domain of influence of $(p+2)$ knots. B-spline basis functions of degree one and degree two over uniform knot vector are shown graphically below in figures (1) and (2).

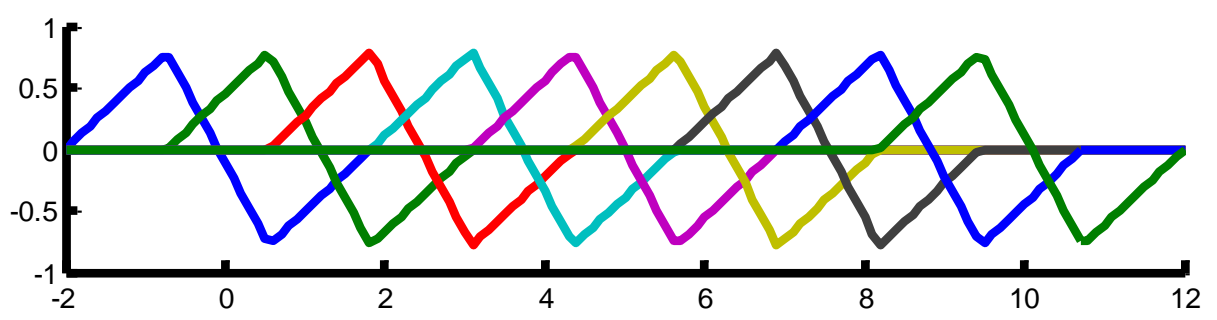

Figure 3: First derivative of second degree B-spline basis function with uniform Knot vector $\mathrm{X}=\{-2,-1,0,1,2,3,4,5,6,7,8,9,10,11,12\}$ 


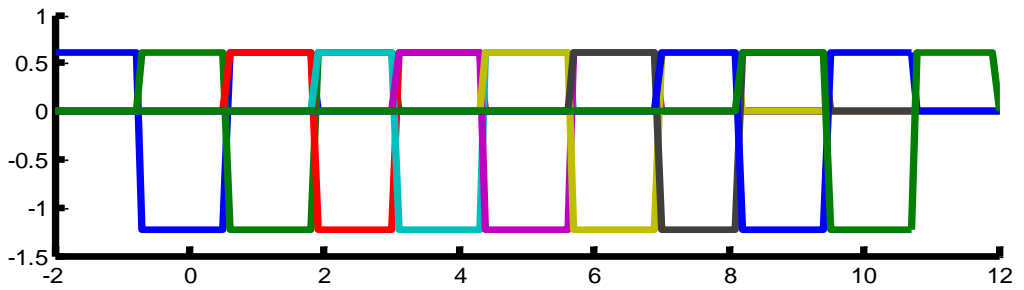

Figure 4: Second derivative of second degree B-spline basis function with uniform Knot vector $\mathrm{X}=\{-2,-1,0,1,2,3,4,5,6,7,8,9,10,11,12\}$

\subsection{B-spline collocation method}

Collocation method is widely used in approximation theory particularly to solve differential equations .In collocation method, the assumed approximate solution is made it exact at some nodal points by equating residue zero at that particular node. B-spline basis functions are used as the basis in B-spline collocation method whereas the base functions which are used in normal collocation method are the polynomials vanishes at the boundary values. Residue which is obtained by substituting equation (2) in equation (1) is made equal to zero at nodes in the given domain to determine unknowns in (2).Let $[a, b]$ be the domain of the governing differential equation and is partitioned as $X=\left\{a=x_{0}, x_{1}, x_{2} \ldots \ldots . . x_{n-1}, x_{n}=b\right\} \quad$ with equal length $h=\frac{b-a}{n}$ of $n$ sub domains. The $x_{i}{ }^{\prime} s$ are known as nodes, the nodes are treated as knots in collocation B-spline method where B-spline basis functions are defined and these nodes are used to make the residue equal to zero to determine unknowns $C_{i}$ 's in (2).two extra knot vectors are taken into consideration beside the domain of problem both side when evaluating the second degree B-spline basis functions at the nodes.

Substuiting, the approximate solution (2) and its derivatives in (1).

$$
\begin{gathered}
\frac{d^{2} U^{h}}{d x^{2}}+k_{1} P(x) \frac{d U^{h}}{d x}+k_{2} Q(x) U^{h}=R(x) \quad \text { i.e. } \\
\sum_{i=-2}^{n-1} C_{i} N^{\prime \prime}{ }_{i, p}(x)+k_{1} P(x) \sum_{i=-2}^{n-1} C_{i} N^{\prime}{ }_{i, p}(x)+k_{2} Q(x) \sum_{i=-2}^{n-1} C_{i} N_{i, p}(x)=R(x)
\end{gathered}
$$

Equation (5) which is valuated at $x_{i}{ }^{\prime} s, \mathrm{i}=0,1,2, \ldots . \mathrm{n}-1$ gives the system of $(\mathrm{n}-1) \times(\mathrm{n}+1)$ equations in which $(n+1)$ arbitrary constants are involved. Two more equations are needed to have $(n+1) \times(n+1)$ square matrix which helps to determine the $(n+1)$ arbitrary constants. The remaining two equations are obtained using

$$
\sum_{i=-2}^{n-1} C_{i} N_{i, p}(a)+\varepsilon \sum_{i=-2}^{n-1} C_{i} N_{i, p}^{\prime}(a)=\mathrm{d}_{1} \sum_{i=-2}^{n-1} C_{i} N_{i, p}(b)+\sum_{i=-2}^{n-1} C_{i} N_{i, p}(b)=\mathrm{d}_{2},
$$

Now using all the above equations (5), (6) i.e. $(n+1)$ a square matrix is obtained which is diagonally dominated matrix because every second degree basis function has values other than zeros only in three intervals and zeros in the remaining intervals, it is a continuing process like when one function is ending its effect in its surrounding region than other function starts its effectiveness as parameter value changing. In other words, every parameter has at most under the three $(\mathrm{p}=2)$ basis functions. The systems of equations are easily solved for arbitrary constants 
$\mathrm{C}_{\mathrm{i}}$ 's. Substuiting these constants in (2), the approximation solution is obtained and used to estimate the values at domain points.

\section{NUMERICAL EXPERIMENTS}

Numerical Example 1.

The effectiveness of the present method is demonstrated by considering the various examples Example 1: Consider linear singularly perturbed boundary value problem given

$$
-\varepsilon U^{\prime \prime}+U(x)=-\cos ^{2}(\pi x)-2 \varepsilon x^{2} \cos (2 \pi x), \quad 0<x \leq 1, \quad U(0)=0 \quad, U(1)+\varepsilon U^{\prime}(1)=1
$$

The exact solution is

$U(x)=\left(\left(\exp (-(1-\mathrm{x}) /(-1 / \sqrt{\varepsilon}))+\exp (-\mathrm{x} . /(-1 / \sqrt{\varepsilon})) /(1+\exp (-1 / \sqrt{\varepsilon}))-\cos ^{2}(\pi \mathrm{x})\right.\right.$

Cubic B-spline collocation is applied to the numerical example 1 and presented the values in the Table 1 at some nodes also included in the same Table 1 exact values at corresponding nodes. It is noticed from the Table 1 that the values which are obtained by present method is almost equal to the values of exact values for different values of $\varepsilon$.This shows that the present method is successfully demonstrated to find the numerical solution of linear singularly perturbed boundary value problem. These values are calculated by considering 101 collocation points .

Table 1: Presents Comparison of present and exact solutions

\begin{tabular}{|l|l|l|l|l|l|l|}
\hline \multirow{2}{*}{$\begin{array}{l}\text { node } \\
\mathrm{s}\end{array}$} & \multicolumn{2}{|c|}{$\varepsilon=1 / 12$} & \multicolumn{2}{c|}{$\varepsilon=1 / 64$} & \multicolumn{2}{c|}{$\varepsilon=1 / 256$} \\
\cline { 2 - 7 } & $\begin{array}{l}\text { Present } \\
\text { Solution }\end{array}$ & $\begin{array}{l}\text { Exact } \\
\text { solution }\end{array}$ & $\begin{array}{l}\text { Present } \\
\text { solution }\end{array}$ & $\begin{array}{l}\text { Exact } \\
\text { solution }\end{array}$ & $\begin{array}{l}\text { Present } \\
\text { solution }\end{array}$ & $\begin{array}{l}\text { Exact } \\
\text { solution }\end{array}$ \\
\cline { 2 - 7 } .01 & -0.0310 & -0.0310 & -0.0759 & -0.0758 & -0.1470 & -0.1469 \\
\hline .5 & 0.3429 & 0.3431 & 0.0365 & 0.0366 & 0.0006 & 0.0007 \\
\hline .99 & -0.0310 & -0.0310 & -0.0759 & -0.0758 & & -0.1470 \\
\hline
\end{tabular}

Numerical Example2

$-\varepsilon U^{\prime \prime}+(1+x) U(x)==-40\left(\mathrm{x}\left(\mathrm{x}^{2}-1\right)-2 \varepsilon\right), \quad 0<x \leq 1, \quad U(0)=0 \quad, U(1)=0$

The exact solution is $U(x)=40 \mathrm{x}(1-\mathrm{x})$

The present method is tested for the numerical example 2 for the different values of $\varepsilon\left(=10^{-5}, 10^{-}\right.$

$7,10^{-9}$ ) with the different boundary conditions. Compared values of present method and exact solution at different various nodes is shown in Table 2.

Table 2: Presents Comparison of present and exact solutions

\begin{tabular}{|l|l|l|l|l|l|l|}
\hline \multirow{2}{*}{$\begin{array}{l}\text { node } \\
\mathrm{s}\end{array}$} & \multicolumn{2}{|c|}{$\varepsilon=10^{-5}$} & \multicolumn{2}{c|}{$\varepsilon=10^{-7}$} & \multicolumn{2}{c|}{$\varepsilon=10^{-9}$} \\
\cline { 2 - 7 } & $\begin{array}{l}\text { Present } \\
\text { solution }\end{array}$ & $\begin{array}{l}\text { Exact } \\
\text { solution }\end{array}$ & $\begin{array}{l}\text { Present } \\
\text { solution }\end{array}$ & $\begin{array}{l}\text { Exact } \\
\text { solution }\end{array}$ & $\begin{array}{l}\text { Present } \\
\text { solution }\end{array}$ & $\begin{array}{l}\text { Exact } \\
\text { solution }\end{array}$ \\
\cline { 2 - 7 } .01 & 0.3960 & 0.3960 & 0.3960 & 0.3960 & 0.3960 & 0.3960 \\
\hline
\end{tabular}




\begin{tabular}{|l|l|l|l|l|l|l|}
\hline .5 & 10 & 10 & 10 & 10 & 10 & 10 \\
\hline .99 & 0.3960 & 0.3960 & 0.3960 & 0.3960 & 0.3960 & 0.3960 \\
\hline
\end{tabular}

\section{CONCLUSIONS}

The B-spline basis functions defined recursively are incorporated in the collocation method and applied the same to second order linear singularly perturbed two point boundary value problem. The effectiveness of the proposed method is illustrated by considering two numerical examples. The solution is compared with exact solution and found to be in good approximation. This method may be applied to different types of second order linear singularly perturbed two point boundary value problem for its efficiency.

\section{REFERENCES}

[1] Hughes, T.J.R., Cottrell, J.A. and Bazilevs, Y. "Isgeometric analysis: CAD, finite elements, NURBS, exact geometry and mesh refinement"', Comput.Methods Appl. Mech. Engg., 194(39-41), pp. 4135-4195 (2005).

[2] David F.Rogers and J.Alan Adams, "Mathematical Elements for Computer Graphics", 2nd ed., Tata McGraw-Hill Edition, New Delhi.

[3] C. de Boor and K. H"ollig. B-splines from parallelepipeds. J. Analyse Math., 42:9915,1982

[4] R.K. Pandey and Arvind K. Singh, On the convergence of a finite difference method for a class of singular boundary value problems arising in physiology, J. Comput. Appl. Math 166 (2004) 553-564.

[5] Geng, F.Z. and Cui, M.G. (2007) Solving Singular Nonlinear Second-Order Periodic Boundary Value Problems in the Reproducing Kernel Space. Applied Mathematics and Computation, 192, 389-398.

[6] Li, Z.Y., Wang, Y.L., Tan, F.G., Wan, X.H. and Nie, T.F. (2012) The Solution of a Class of Singularly Perturbed Two-Point Boundary Value Problems by the Iterative Reproducing Kernel Method. Abstract and Applied Analysis, 1-7

[7] Mohsen, A. and El-Gamel, M. (2008) On the Galerkin and Collocation Methods for Two Point Boundary Value Problems Using Sinc Bases. Computers and Mathematics with Allications, 56, 930-941.

[8] Abdalkaleg Hamad, M. Tadi, Miloje Radenkovic (2014) A Numerical Method for Singular Boundary-Value Problems. Journal of Applied Mathematics and Physics, 2, 882-887.

[9] Joan Goh_, Ahmad Abd. Majid, Ahmad Izani Md. Ismail (2011) Extended cubic uniform B-spline for a class of singular boundary value problems. Science Asia 37 (2011): 79-82

[10] I. J. Schoenberg Contributions to the problem of approximation of equidistant data by analytic functions, Quart. Appl. Math. 4 (1946), 45-99; 112-141.

[11] H. B. curry and I. J. Schoenberg On Polya frequency functions IV: The fundamental spline functions and their limits, J. Anal. Math. 17 (1966), 71-107.

[12] Carl de boor On Calculating with B-plines. Journal of approximation theory6, so-62 (1972) 
[13] M. K. Kadalbajoo, Y.N. Reddy, Asymptotic and numerical analysis of singular perturbation problems (1989),Applied mathematics and computation 1989; 30: 223-259.

[14] M.K. Kadalbajoo and K.C. Patidar, A survey of numerical techniques for solving singularly perturbed ordinary differential equations, Applied Mathematics and Computation 130(2-3) (2002) 457-510. 